\title{
CONSTRUYENDO JUNTOS LOS PROCESOS DE ALFABETIZACIÓN: NÚCLEO TAKINAHAKỸ : UFG, BRASIL
}

\author{
José Guadalupe Mayo Rosado ${ }^{1}$ \\ Maria do Socorro PImentel da Silva ${ }^{2}$
}

\section{RESUMEN}

La finalidad es compartir algunas de las aportaciones académicas que se desarrollan durante el proceso de la formación de los docentes, entorno a la didáctica del aprendizaje y de la enseñanza de la lectura y la escritura, situación que se genera a partir del tema contextual " "Procesos de alfabetización", en una de las etapas del curso (julio de 2017) de la Licenciatura Intercultural de Formación Superior Indígena, programa que imparte la Universidad Federal de Goiás (UFG), de manera particular se pretende mostrar cómo los profesores-estudiantes a partir de sus saberes culturales y de sus prácticas sociales que comparten en forma grupal construyen otros conocimientos que les permiten ampliar el abanico pedagógico sobre la didáctica de la enseñanza de la lectura y la escritura a las niñas y a los niños de sus escuelas (alfabetización y letramento).

PALABRAS CLAVES: Procesos de alfabetización, formación docente, práctica docente.

\section{ABSTRACT}

1 Actualmente cursando el Doctorado en Pedagogía en el Programa de posgrado de la UNAM, en la ciudad de México. Profesor de educación primaria, en el estado de Quintana Roo, México. Ciudad de México CDMX, México. E-mail:josemayo2003@yahoo.com.mx.

2 Doutora em Linguística Aplicada ao Ensino de Línguas pela Pontifícia Universidade Católica de São Paulo. Pós-doutorado em Linguística pela UNI-CAMP. Professora do Núcleo Takinahakỹ de Formação Superior Indígena (NTFSI) e da Pós-Graduação em Letras e Linguística/UFG. Goiânia, GO,Brasil. E-mail:smariapimentel@yahoo.com.br.

3 Con el término "tema contextual" se hace referencia a los saberes, practicas, tradiciones y cultura del escolar, situaciones que deben ser aprovechadas como un medio y recurso de interés para propiciar el conocimiento académico. 
The purpose is to share some of the academic contributions that are developed during the process of teacher training, in the context of the didactics of learning and teaching of reading and writing, a situation that is generated from the contextual theme1 "Processes of literacy", in one of the stages of the course (July 2017) of the Intercultural Degree of Indigenous Higher Education, a program taught by the Federal University of Goiás (UFG), in a particular way aims to show how teachers-students from their cultural knowledge and social practices that they share in group form, they construct other knowledge that allows them to broaden the pedagogical range on the teaching of reading and writing teaching to the girls and the children of their schools (literacy and literacy).

KEYWORDS: Literacy processes, teacher training, teaching practice.

\section{Introducción}

Los procesos del aprendizaje y de enseñanza de la lectura y la escritura, encaminados hacia la alfabetización, es un acto de compromiso, de interés y reto, tanto para los docentes como para los escolares, "enseñar a leer y escribir es un desafío que trasciende ampliamente la alfabetización en un sentido estricto" (Lerner, 2004: 25), situación que debe, además, girar con claridad, al por qué y al para qué, entre otras premisas. El artículo comparte cómo los profesores - estudiantes de diferentes pueblos originarios de Brasil, durante el proceso de su formación en la licenciatura, a partir del análisis de sus prácticas docentes, juntos construyen, tanto la concepción de la alfabetización, como estrategias didácticas para propiciar en los escolares de sus escuelas, el logro de sus objetivos pedagógicos.

Las interacciones, así como las relaciones inter-étnicas, la socialización de saberes y conocimientos docentes en función de la enseñanza de la lectura y escritura fueron posible mediante situaciones dialógicas (Mercado, 2012: 35) propiciadas por la profesora del grupo. Teniendo en cuenta las características que dan bases epistemológicas y sustento teórico a la estructura del programa de la licenciatura y en lo especifico, a la práctica académica mediante la cual se desarrolla el tema contextual "Procesos de alfabetización", se hace un acercamiento a las aportaciones que distinguen a la teoría de la complejidad, Morín (2008), así como también se abordan 
contribuciones de autores como Davini (2016), Schön (2010), Freire (2016, 2013, 1996), entre otros.

La participación como un integrante más del grupo permitió un acercamiento mayor y confianza entre los profesores-alumnos, situación que se aprovechó para abordar conversaciones, que se convirtieron en pequeños diálogos sobre la temática. El contexto me permitió estar como observador participante, Taylor (1996), se hizo el registro de actividades en la bitácora, el procesamiento y análisis de los datos, se abordan teniendo en consideración las orientaciones metodológicas que comparte tanto Tarrés (2013), Bertely (2000) y Strauss (1967) principalmente.

\section{En el camino de la alfabetización}

La formación de los profesores se da a través del curso de la Licenciatura Intercultural de Formación Superior Indígena, programa que imparte la Universidad Federal de Goiás (UFG) ${ }^{42}$, con sede en Goiania capital del estado, en Brasil ${ }^{53}$, aunque esta licenciatura es muy reciente en la universidad (2007), existen otras universidades Brasileiras $^{64}$ que presentaron proyectos contemporáneos a la de otros países latinoamericanos (Bolivia 1994, Ecuador 1986, México 1984); el curso se desarrolla a través del núcleo Takinahaky, el plan de estudios tiene sus particularidades, ya que se desarrolla en función

4 El programa de la licenciatura funciona de manera independiente en comparación con el resto de las licenciaturas adscritas de acuerdo a las áreas de estudios a las diversas facultades de la Universidad Federal de Goiás (UFG); a finales del año 2016 se realizó el primer proceso de selección para ingreso y dio inicio en el año 2007.

5 Brasil, país ubicado en Sudamérica, caracterizado entre otras cosas por ocupar el quinto lugar en extensión territorial y demografía poblacional, es el territorio con mayor cantidad de cuerpos hídricos y espacio habitado por más de 220 pueblos originarios $^{3}$ (Santos, 2006:12), esta riqueza cultural le permite ser único en cantidad de pueblos amerindios, pero también implica mayor responsabilidad para atender desde los diferentes servicios públicos los derechos que cada uno de los ciudadanos demanda en un plano de igualdad.

6 En Brasil a finales de 1990 las universidades que ofrecieron programa de licenciaturas indígenas fueron: UNEMAT Universidad Estadual de Mato Grosso, UFR Universidad Federal de Roraima, UFMG Universidad Federal de Minas de Gerais, entre otras. 
tres conceptos claves: la interculturalidad, la transdisciplinariedad y la contextualidad, esta postura curricular es un llamado a las demandas de profesores convencidos de su cultura, tradiciones y saberes, en donde además, la lengua materna es uno de los pilares para promover la herencia cultural y con ello garantizar los saberes de los pueblos a las nuevas generaciones, sin perder la esencia de los fines de la educación.

Cabe señalar que es de gran interés pedagógico conocer cómo el tema contextual "Proceso de alfabetización", contribuye en la formación de los docentes, se pretende compartir como en el desarrollo de las actividades didácticas, los profesores - estudiantes, socializan saberes cotidianos que forman parte de su riqueza cultural y cosmológica, situaciones que son encauzados pedagógicamente al proceso de la alfabetización.

En este sentido y tomando en cuenta la función e importancia que tiene el aprendizaje de la lectura y la escritura en todos los contextos sociales, pero que además en la actualidad existe preocupación e interés constante tanto por educadores e investigadores desde diversas disciplinas por la temática de la alfabetización en los escolares, la profesora coordinadora del tema contextual "Procesos de alfabetización”, nos permite adentrarnos a la forma de cómo se genera el ambiente académico en la formación de los profesores.

Enseñar a leer y escribir, no es tarea fácil, es preciso estar involucrado en conocimientos teóricos que van desde la psicología, la pedagogía, la didáctica y su enseñanza, entre otros, así como dominio del arte de la docencia en nivel básico (vocación), tener bien clara la meta, sobre porqué y para qué, por otra parte, discriminar entre los métodos de enseñanza para la lectura y la escritura las ventajas y las desventajas que ofrecen, sobre estos existen una variedad, mismo que se clasifican en dos grandes grupos los métodos sintéticos y globales, pero para algunos profesores va más allá de lo planteado pues implica conocer y dominar la lengua materna de los escolares así como conocer sus tradiciones y costumbres, en otras palabras, ser y estar integrado en la comunidad.

La preocupación por comprender los procesos de aprendizaje de la lectura y la escritura, generó en algunos países: México, Argentina, España, Brasil, que a finales de las décadas de los 70’ del 
siglo $\mathrm{XX}^{75}$, iniciaran las primeras investigaciones sobre la temática de la lectura y la escritura, a la fecha, existen muchos estudios e investigaciones relacionados a la psicogenésis de la escritura, aportaciones sustentadas a partir de las teorías de Piaget, entre las que destacan los estudios de: Ferreiro (1983, 1985, 1992...2014), Ferreiro y Teberosky (1979), Ferreiro y Palacios (1982), Teberosky (1985, 2003), Nemirosky (1999), Soares (1998, 1989, 1986), Palacios (1982), entre otros, mismas que coinciden en sus planteamientos y en los procesos del aprendizaje del sujeto en función de la escritura, este grupo de investigadoras desde una postura del campo de la psicología, sostienen (entre otras aportaciones) la idea que el aprendizaje del sistema de la escritura y de la lectura entre las niñas y los niños es un proceso que corresponde a patrones cognitivos casi similar y existen diversos factores que interfieren (ambientes alfabetizadores), sin embargo existen pocos estudios en aquellos contextos sociales y culturales en donde las tradiciones y los saberes cosmológicos son factores que interfieren en el proceso de la alfabetización, ejemplo de ello, son las practicas educativas que se da en los pueblos amerindios de Brasil y países con población originaria.

En este sentido y teniendo en consideración que en los procesos educativos la alfabetización (aprendizaje de la lectura y la escritura) en la lengua materna, es un derecho y propósito que todo escolar debe alcanzar, pues el aprendizaje de estos son herramientas elementales para entender las prácticas sociales comunicativas del lenguaje escrito, por una parte y por otra, es una herramienta que se demanda en cualquier contexto social, sin importar el lugar donde se ubique el escolar, le permite el aprendizaje de nuevos conocimientos académicos de los grados sucesores; por lo que se considera de suma importancia conocer cómo aprenden los escolares este proceso de alfabetización en función de la formación de los profesores y

7 Palacios, siendo directora de la Dirección General de Educación Especial, preocupada por la situación que se presentaban en muchas escuelas al tener mucho rezago educativo en función de la falta de aprendizaje de la lectura y la escritura en los escolares, junto con Emilia Ferreiro diseñaron algunas estrategias pedagógicas y con sus equipos de trabajos realizaron una serie de pruebas en diferentes lugares de México (Distrito Federal, Monterrey y Mérida), el trabajo fue laborioso pero les permitió fundamentar y proponer las primeras bases, sus investigaciones denominadas "Análisis de las perturbaciones en el proceso del aprendizaje de la lecto-escritura", integrado en 5 fascículos; 
entorno a los saberes culturales y comunitarios, en este sentido, "Cuando hablamos del profesor nos estamos refiriendo a alguien que se sumerge en el complejo mundo del aula para comprenderla de forma crítica y vital, implicándose afectiva y cognitivamente en los intercambios inciertos, analizando los mensajes y redes de interacción, cuestionando sus propias creencias y planteamientos proponiendo y experimentando alternativas y participando en la reconstrucción permanente de la realidad escolar" (Schön, 2010: 89)

En lo particular y en relación al tema contextual "Procesos de alfabetización" es de vital importancia difundir desde estos escenarios algunas de las experiencias que comparten profesores y profesoras entorno al aprendizaje y la enseñanza de la lectura y escritura como un todo, desde el contexto de cada pueblo, cabe señalar que las acciones involucran a toda la comunidad, además del profesor y de los escolares, es un proceso complejo, Morín hace referencia sobre la complejidad "cuando no se pueden separar los componentes diferentes que constituyen un todo (como lo económico, lo político, o sociológico, lo psicológico, lo afectivo, lo mitológico) y cuando existe tejido interdependiente, interactivo e Inter retroactivo entre las partes y el todo, el todo y las partes (2008: 14), en este sentido los procesos de aprendizaje y de enseñanza de la lectura y escritura no son acciones independientes y aisladas, sino que éstos tienen que tener de fondo, un tema de interés y de importancia al que se tiene que encausar pedagógicamente, vemos entonces que los procesos de alfabetización no son aislados, sino que éste es una tarea que se tiene que construir juntos, profesor -alumno, ambiente y agente alfabetizador.

En este sentido y con el interés de compartir una pequeña parte de lo que contribuye al trayecto formativo de los docentes de algunos de los grupos originarios del país de Brasil, a groso modo se describen actividades realizadas en la semana del trabajo de campo del 17 al 22 de julio de $2017^{86}$, el registro de la práctica docente en la bitácora es uno de los instrumentos que dan pauta al sustento epistémico empírico de los profesores en su formación profesional, además de ser un testimonio que da solidez al presente trabajo, desde

8 La licenciatura se caracteriza por estar enfocada a profesores en servicios por lo que el sistema es semipresencial, distribuida en los periodos de recesos escolar o vacaciones como son: las semanas de enero a febrero y cinco semanas de los meses que van de julio a agosto. 
esta perspectiva y como bien lo señala Schön (2010) reflejar el transitar académico de los profesores por la universidad como un espacio que contribuye en la formación profesional de la docencia y que les permite desenvolverse con mayor conciencia en el papel docentes en sus comunidades, sin olvidar que "Las practicas docentes están integradas por un amplio abanico de capacidades concretas relacionadas con los ejes centrales de la acción profesional, cualquiera que sea el contexto escolar especifico en el que el docente particularmente se desempeñe" (Davini, 2016:83).

La etnografía de aula emprendida, permitió entender, cómo los profesores de los pueblos originarios de Brasil construyen juntos su formación docente en función de la temática citada, también me brindó la oportunidad de conocer las estrategias didácticas que desarrollan en sus prácticas docentes, lo que permite sostener que es un tema poco abordado por estudiosos desde el campo de la pedagogía.

De igual manera, esta oportunidad de hacer investigación en el aula mediante la convivencia entre iguales, pero diferentes, en la vía de la formación docente, permite ver las relaciones, los saberes que se comparten y los resultados que se generan al construir juntos nuevas estrategias didácticas sobre el aprendizaje de la alfabetización, para poder comprender las prácticas pedagógicas en las comunidades amerindias, en función de estos resultados generar ideas didácticas que permitan dar alternativas pedagógicas que apuntalen a democratizar la educación en los países étnicamente diferenciados.

\section{El objetivo}

Documentar la práctica docente del tema contextual "Procesos de alfabetización", para conocer cómo los alumnos de la Licenciatura Intercultural de Formación Superior Indígena construyen juntos concepciones sobre la alfabetización, así como también diseñan estrategias didácticas para la enseñanza de la lectura y la escritura.

\section{Objetivos particulares}

Relacionar las estrategias didácticas desarrolladas en el tema contextual "Procesos de alfabetización", y las formas de concebirlas 
por los alumnos, para comprender los procesos de formación como docentes de la Licenciatura en Educación Intercultural.

Analizar de qué manera los estudiantes - profesores entiende el proceso de alfabetización y lo que conlleva implícitamente el proceso de aprendizaje y de la enseñanza.

\section{Aprendizaje de la lectura y la escritura}

La práctica académica del tema contextual, "Procesos de alfabetización" en la Licenciatura del programa de la UFG, es parte un "curriculum" que está en constante construcción a partir del compromiso profesional de la profesora a su cargo, el análisis de paradigmas pedagógicos a la par de la realidad sociocultural y geográfica de los estudiantes que son elementos considerados como principales para el desarrollo de la misma.

Leer y escribir son procesos complejos, también es una necesidad predominante en casi todas las sociedades, en algunas por ejemplo, no se puede acceder a un empleo si no se cuenta con niveles educativos de nivel medio, y para el logro de éste, es necesario cursar los grados escolares en la educación básica, lo cual demanda de lo fundamental, el dominio y desarrollo de la lectura y la escritura, de esta manera podemos observar lo elemental y la importancia de su aprendizaje en los escolares, son por lo tanto, "un artefacto cultural que es preciso manejar con soltura para alcanzar el éxito social, académico y profesional en sociedades como la nuestra, en las que el acceso al conocimiento y la información están altamente mediados por ella", (Rodríguez, 2017: 19). El planteamiento señalado, es un discurso que encaja en un gran porcentaje de sociedades modernas, en las poblaciones que predominan ante las originarias de una región, de un país, pero, serán acaso las mismas necesidades y prioridades que se generan en los niños y en las niñas de los pueblos originarios, el denominar común y al que no se puede negar, es que, es un proceso complejo, más de fondo tiene otros fines como bien lo señala Pimentel $(2016,53)$ "las funciones sociales del aprendizaje de la lengua escrita tiene como finalidad... documentar saberes, (4) fortalecer la memoria; (5) incrementar la transmisión de saberes tradicionales de una generación a otra", entre otros.

Ahora bien, pareciera fácil aprender a leer y escribir, pero esto es un proceso que requiere además de su tiempo, ambientes y 
espacios en donde se reflejen los diferentes usos de la escritura y destinatarios, profesores que estén convencidos de la importancia de su función en la enseñanza de la lectura y la escritura, amén del dominio didáctico de la enseñanza, conocer la diversidad de elementos o factores que pudieran incidir en el proceso del aprendizaje de la niña y del niño, partiendo como base del conocimiento en sí de las características de los infantes, el contexto socio cultural y geográfico, en el que está inmerso, tener con claridad el rumbo e importancia de la alfabetización, entre otros,.

En relación a los procesos de la enseñanza de lectura y la escritura, se puede decir que, en el devenir histórico y desarrollo de la educación, en ciertas sociedades han perpetuado de generación en generación algunas de las didácticas de la enseñanza de la lectura y de la escritura, sin retroceder mucho al pasado de éstas, aún son conocidos métodos muy antiguos, como lo serían los Silabarios, con los cuales, y por muchas pero muchas décadas han aprendido a leer y a escribir millones de niñas y de niños, además ha funcionado como inspiración en la construcción de otros métodos didácticos ${ }^{97}$ como: el método de mantilla, el método fonético, el método de palabras normales, entre otros (Barbosa, 1988, Rincón, 2003, Rébsamen, 1968), que son variantes de entre ellos, pero a veces conocidos con otros nombres; este planteamiento nos da un acercamiento a como también se dan los momentos que permiten construir ideas que conlleven hacia formas de ser más accesible el aprendizaje de la lectura y la escritura.

Un avance significativo reflejado en la concepción de la escritura y su enseñanza inició a finales de los 70' del siglo pasado, investigaciones de Ferreiro y Teberosky (1979) en Argentina, Ferreiro y Palacios (1982) en México, paralelamente Teberosky (1980) en España, luego Nemirosky (1984) en Argentina, permitieron un pequeño giro entorno al sentido y la forma de concebir de la

9 Uno de los factores que generaron la creación de la diversidad o adecuación de los métodos didácticos en México fueron sin duda alguna la preocupación de los profesores al enfrentarse a la diversidad cultural, contextual y geográfica ya que si bien la escuela rural mexicana tenía un plan educativo definido y ambicioso entre las cuales la castellanización también era de su interés por lo que la aplicación o uso de algunos métodos didácticos no eran efectivos ante la población escolar indígena, lo que propiciaba la preocupación en los docentes y con ello la adaptación o construcción de otros métodos didácticos. 
escritura y la lectura, fundamentadas en las aportaciones de Piaget. Un dato de interés es que estas investigadoras, si bien emprendieron desde países diferentes sus investigación implícitamente trabajan juntas, conforman un equipo que investigan todo lo relacionado a la alfabetización, sus aportaciones han permitido a los docentes, entre otros saberes, conocer desde sus posturas teóricas, procesos y factores que interfieren en el aprendizaje en función de la lectura y de la escritura (psicogénesis), con ello dar más elementos pedagógicos a la función docente para abordar desde estos planteamientos la enseñanza de la escritura y la lectura.

De igual manera estas aportaciones dieron pauta a la construcción y ajuste de nuevas definiciones y formas de comprender los conceptos de la lectura y la escritura desde posturas teóricas casi similares, así por ejemplo Ferreiro plantea que la escritura "puede ser conceptualizada de dos maneras muy diferentes y, según sea el modo en que se la considere, las consecuencias pedagógicas difieren drásticamente. La escritura como una representación del lenguaje o como un código de transcripción grafico de las unidades sonoras" $(1997,13)$.

Cabe hacer mención que el grupo de investigadoras citadas proceden de un mismo campo de estudio, además como bien lo indica Ferreiro y Teberosky $(1979,285)$ "Hemos usado el marco conceptual de la psicología genética para elaborar nuestra hipótesis. Este libro es la reiterada prueba de la pertinencia y fruto de la teoría piagetana para la comprensión de los procesos de la adquisición del conocimiento de un dominio no estudiado por el propio Piaget", las aportaciones de estas autoras respecto al libro "Los sistemas de escritura en el desarrollo del niño" no plantea métodos didácticos para la enseñanza de la lectura y la escritura, sin embargo a partir de las publicaciones han generado un revuelto en los docentes, por otro lado, la postura de este grupo de investigadoras (citadas con anterioridad), son partidarias de la idea, que la enseñanza de la lectura y la escritura debe partir de lo general a lo particular, en cierto grado, están en desacuerdo en los usos de los métodos para la enseñanza de la lectura y la escritura, sobre todo de los llamados 
métodos de marcha sintética ${ }^{108}$. De la misma manera opinan que se aprende a leer leyendo y a escribir escribiendo.

Otra investigadora reconocida en Brasil por sus aportaciones a la temática de la alfabetización es la profesora Magda Soares ${ }^{119}$ (1998, 1989, 1986), su trayectoria en función de la enseñanza de la lectura y de la escritura y como ella lo denomina aprendizaje inicial de la lengua escrita, en su tesis principal "aprendizaje inicial de la lengua escrita" explica la implicación de dos procesos como son: alfabetización y letramento.

El proceso de alfabetización es llanamente la adquisición del sistema de alfabeto, los pasos de cómo transformamos nuestro idioma en letras y fonemas (grafías) hasta lograr dominarla, que sería el letramento (uso de la lectura y escritura en los diversos contextos), pero además de éste, implica del desenvolviendo de habilidades de uso de la tecnología escrita, tanto uno y para el otro es también necesario ambientalizar los espacios (aulas escolares) con libros, trabajos de los alumnos etc.

El letramento es por lo tanto, producir un texto con sentido y destinatario, hacer uso de la escritura, redactar un oficio, hacer una carta con los elementos que la caracterizan, etc.

La profesora Soares también argumenta que se aprende a leer leyendo y a escribir escribiendo, es necesario partir de temas reales y vivenciales de los escolares para que descubran el sentido de los procesos de representaciones, de igual manera es indispensable ambientalizar los espacios escolares con textos, publicaciones y

10 Inicialmente, el aprendizaje de la lectura y escritura es una cuestión mecánica; se trata de adquirir la técnica de descifrado del texto. Porque la escritura se concibe como la transcripción gráfica del lenguaje oral, como su imagen (imágenes más o menos fieles según los casos particulares), leer equivale a decodificar lo escrito en sonido... $(1979,19)$

11 Possui graduação em Letras Neolatinas pela Universidade Federal de Minas Gerais (1953) e doutorado em Didática também pela Universidade Federal de Minas Gerais (1962). Atualmente é membro da Associação Nacional de Pós-Graduação e Pesquisa em Educação, membro de comitê assessor do Conselho Nacional de Desenvolvimento Científico e Tecnológico, consultora de Coordenação de Aperfeiçoamento de Pessoal em Nível Superior, conselheira da Communautée économique éuropéene, professora titular da Universidade Federal de Minas Gerais, da Universidade Federal de Minas Gerais. Tem experiência na área de Educação, com ênfase em ensino-aprendizagem. 
libros. En este sentido y como lo plantea Lerner (2004), participar en la cultura escrita supone apropiarse de una tradición de lectura y escritura, supone asumir una herencia cultural que involucre el ejercicio de diversas operaciones con los textos y la puesta en acción de conocimientos sobre las relaciones entre los textos... (25).

Se puede decir que las propuestas didácticamente están encaminadas a propiciar la alfabetización, pero no funcionan de forma aisladas, no solo por el hecho de que los escolares tengan acceso a los textos, se alfabeticen, sino que es necesario propiciar el interés para que los escolares se acerquen a explorar dichos materiales, a conocer y consultar, entonces es donde entra al escenario del docente, su creatividad para generar las condiciones que conlleven al escolar el acercarse a los materiales, el proceso de alfabetización es entonces una tarea que se tiene que realizar en equipo, juntos alumnos docente, docente alumnos, pues es necesario aprovechar el interés, el contexto y los estilos o ritmos de aprendizaje del escolar, y tener en cuenta las áreas de oportunidad, pero a su vez el escolar demanda al profesor una atención personalizada, propicia las condiciones para integrarse a trabajos entre pares o en grupales.

Sin embargo y en este sentido, teniendo en cuenta que aún en la actualidad no todos los infantes tienen acceso a libros, folletos o publicaciones y que en muchas comunidades rurales y marginadas tampoco existen las cantidades suficientes de ambientes alfabetizadores (carteles, anuncios etc.) que permitan el contacto a las letras para poder emprender las opiniones ya mencionadas, leer leyendo, escribir escribiendo, por lo que esto permite comentar y a la vez se coincide con lo que argumenta Toboada (1997) "existen algunos puntos que merecen ser analizados desde la concepción de la lectoescritura y sus vínculos con el constructivismo de Piaget", además "por limitarse a considerar los conocimientos previos de los y las niñas, sin motivar la activa participación y profundización en la vida cultural de sus pueblos" (2015: 16), comenta y agrega Carrillo (2009), "en los procesos del aprendizaje de la lectura y la escritura, no se puede generalizar las formas de enseñar, ni se pueden esperar resultados grupales en tiempos determinados", el aprendizaje no sólo se reproduce en sesiones con instrucciones específicas, es necesario tener presente que cada niño posee características totalmente diferentes en los estilos y ritmos de aprendizaje, ante la realidad imperante en los diferentes contextos socioculturales y geográficos de los escolares. 
Existen otras líneas de investigaciones en función de los procesos de aprendizaje de la lectura y de la escritura, me refiero a los trabajos realizados por algunos estudiosos ${ }^{1210}$ quienes han reorientado y enfocados sus temas de estudios en los procesos de la enseñanza y del aprendizaje en los escolares de los pueblos originarios, desde sus contextos sociales culturales y cosmológicos, compartiendo que existen estilos $\mathrm{u}$ formas alternas a las aportaciones de las teorías psicológicas descritas con anterioridad, los resultados de estos estudiosos permiten relacionarlas a los sustentos de la teoría socio - cultural.

Ejemplo de ello son los trabajos de Gasché (2009), Bertely (2008), Pimentel (2017, 2016, 2007), entre otros, tienen la intención de compartir formas alternas a las tradicionales e impuestas practica docentes, en el caso particular de los dos primeros autores, los procesos de sus investigaciones aterrizan en el conocido Método Inductivo Intercultural, en voz de uno de sus protagonista comenta, "va un paso más allá; nos exige no solo quedarnos en el conocimiento previo del niño, sino que nos demanda participar de la vida cultural de la comunidad y profundizar en ella"1311.

Como se ha planteado hasta aquí, las producciones de las investigadoras citadas nos pueden servir de ejemplo, sobre la importancia del trabajo en colegiado, permite enfrentar con mayor solidez los retos y lograr con mayor facilidad los objetivos, en los párrafos siguientes veremos que también los procesos del aprendizaje y de la enseñanza se pueden lograr en equipo.

En esta sintonía, veremos cómo los estudiantes - profesores, juntos construyen otras formas de concebir los proceso de alfabetización, además de socializar sus estrategias didácticas, en función de las experiencias de sus contextos socio - culturales, en

12 En este caso, existen publicaciones de artículos propuestos: Gashé (2008, 2009,) Podestá (2006), Bertely (2009, 2012, 2016) de igual manera, cabe señalar que el CIESA, unidad central (ciudad de México) emprendió un proyecto de investigación enfocado en el contexto intercultural del cual se orientó en trabajos anteriores y realizados en la Amazonía peruana por Jorge Gasché, "Método Inductivo Intercultural," en México el equipo era coordinado por María Bertely...

13 Este comentario se extrajo de una plática la Dra. María Bertely, realizada en la ciudad de Goiás, Brasil, la Dra. fue homenajeada en el III Congreso Internacional de la REF FIAL, celebrado del 26 al 29 del mes de julio del año 2017. 
algunas de las veces derivadas de la tradición oral: aprendizaje y enseñanza de los cantos, el aprendizaje y enseñanza del respecto y cuidado hacia la naturaleza, el aprendizaje y enseñanza sobre la vida de la fauna y la flora, el aprendizaje y enseñanza de la propia cosmovisión y las múltiples manera de entrar en relación en ella, entre otros, situaciones que son catalogadas como temáticas, por lo tanto, conforman actitudes, valores y habilidades que entran en juego en el aprendizaje de los escolares en relación a la adquisición de la lectoescritura ${ }^{1412}$, centradas a la realidad y a su contexto, son actividades de la vida cotidiana de los escolares, por lo tanto son motivo de desarrollarse pedagógicamente, teniendo en cuenta que debe ser en la lengua materna.

Situación que las investigaciones de las primeras autoras citadas poco han abordado e involucrado en sus proyectos, en cambio, podría decirse que el interés de Pimentel ${ }^{1513}$, radica en defender la importancia de la enseñanza de la lectura y la escritura en las lenguas indígenas, por diversos motivos, pues la enseñanza de la lectura y de la escritura en la lengua materna va más allá de lo que han venido considerando algunos investigadores, así por ejemplo, una situación a la que hace frente, Pimentel da Silva, es que, "las lenguas indígenas son consideradas como lenguas de menor prestigio social, económico, político y epistémico, realidades que pone a sus hablantes sentimientos de inferioridades... (Pimentel da Silva, 2016:52), en este sentido vemos como la profesora empoderada del saber y de la práctica docente enfrenta la defensa de los pueblos, a través de la pedagogía, que pocos otros investigadores asumirían, y como muy bien lo argumenta Davini "Cualquier profesión se funda en un cuerpo de conocimientos y de algunos criterios y reglas de acción práctica. La docencia no constituye una excepción: se apoya en un espacio estructurado de conocimientos y criterios y estrategias de acción que, aunque van evolucionando con el tiempo, pueden analizarse en sí mismos" $(2016,21)$.

14 Al respecto existen pocas investigaciones en función de los procesos de los aprendizajes y de enseñanza de la lectura y la escritura en contextos interculturales (Gaiche, 2008; Bertely, 2009).

15 Se utiliza la palabra de interés, debido a que ha sido una de las características de profesional de la profesora que ha manifestado a lo largo de tu trayectoria académica en función del tema contextual "Procesos de alfabetización", mismo que tiene de base y parte de la lengua materna. 
Las aportaciones de las investigadoras abordadas en los primeros párrafos, de manera general y a groso modo, da un referente sobre lo que juntas vienen haciendo, sustentadas en el campo de la psicología, en las líneas sucesivas se procura socializar el desarrollo del tema contextual "Procesos de alfabetización", y la participación de los profesores en su proceso de formación docente.

Los planteamientos se generan desde posturas pedagógicas "propias", muy en lo particular de la profesora a cargo del desarrollo del curso, pues ella, a partir de las experiencias y realidades de los escolares de las diversas comunidades originarias de Brasil ha logrado reorientar la pedagogía, manifestada a través de las actividades académicas, que le permiten socializar desde esas realidades contextualizadas otras concepciones del procesos del alfabetización, como bien lo detalla en su programa del periodo escolar 2017, "la alfabetización como un proceso más amplio de aprendizaje de la lectura y de la escritura. En el contexto social del niño y su relación con el mundo de la escritura" ...

Como se ha mencionado, las prácticas académicas convertidas en experiencias, le permiten argumentar que "La concepción de alfabetización es un proceso ante el cual se debe tener en cuenta la finalidad cómo coadyuvar en el desarrollo del escolar si deprenderse los saberes de la realidad y en este sentido, las estrategias didácticas aplicadas deben de proporcionar el desarrollo potencial del escolar y habilidad lingüística" ${ }^{1614}$, aspecto que se debe tener como base para la enseñanza de la lectura y de la escritura, no se puede y ni es válido pretender la enseñanza de la lectura y la escritura desde una imposición haciendo uso de estrategias didácticas poco comprensibles para los escolares.

Los planteamientos que Pimentel sostiene en sus prácticas académicas durante los procesos de formación en los docentes indígenas se han construido además de la realidades pedagógicas que le ha tocado convivir en los diversos pueblos étnicos del Brasil, en las aportaciones teóricas en las que figuran Freire $(2016,2013$, 2011, 2003, 1994) y Vigostky $(1995,1984,1979,1972)$, respecto a la teoría sociocultural, coincide con lo que se plantea (Vigotsky, 1979) "el lenguaje escrito es un artefacto cultural que constituye un sistema simbólico, y que representa en gran medida al lenguaje oral,

16 Párrafo tomado del programa del tema contextual "Procesos de alfabetización" (2017), realizado por la profesora. 
otro sistema simbólico más primario que a su vez representa a la realidad".

Cabe señalar que de los diversos textos de Vigotsky $(1995,1984,1979)$ se puede distinguir, la importancia de hacer pasar a los niños por sistemas simbólicos primarios, como el lenguaje oral, el gesto o el dibujo, para llegar a comprender el potencial simbólico del lenguaje escrito, "también desde esta corriente se ha resaltado el valor de la narración como una forma de facilitar la comprensión del mundo físico y social a los niños, se aboga por utilizar esta forma de expresión como un medio para ayudarles a manifestar sus pensamientos e ideas" Bruner (1988; 1991) citado por Rodríguez (2017). Planteamientos que coinciden con las prácticas académicas de la profesora.

De esta manera se puede evidenciar que el tema contextual "Procesos de alfabetización" impartido en el curso la licenciatura citada y diseñado por la profesora Pimentel da Silva, es el resultado multifactorial y transdisciplinar, el cual también se ha venido construyendo en torno a la reflexión de varios campos de conocimientos "propios de la educación indígena como lo es la lingüística, psicolingüística, antropología, entre otros ${ }^{1715 ",}$, saberes que contribuyen a la creación de nuevas posturas en el desarrollo de la alfabetización del niño y de la niña indígena.

La interacción textual de la profesora con los investigadores citados y la práctica docente en diversas aldeas de los grupos étnicos ha contribuido a un estilo propio de aprender y enseñar los procesos de alfabetización, como bien lo dice Mercado (1994), "los saberes docentes son pluriculturales, históricos y socialmente construidos", en este sentido, y como se ha mencionado en párrafos arriba, la profesora retoma y hace uso de las aportaciones pedagógicas del pensamiento Freiriano, con la intención demostrar que en los procesos de alfabetización debe de ser "la escuela centrada en la palabra" (Freire, 2001: 86), proceso denominado por la profesora como "metodología contextualizada" por lo que se deben realizar o construir estrategias didácticas desde el interés de los escolares pautados por los saberes y prácticas comunitarias.

17 Párrafo tomado del programa del tema contextual "Procesos de alfabetización” (2017), realizado por la profesora. 


\section{La alfabetización inicial en vía de aprendizaje en}

\section{comunidades diversas: los pasos}

En las siguientes líneas se trata de ilustrar el trabajo de campo realizado desde la perspectiva de la etnografía escolar (Tarrés, 2013; Rockwell, 2009; Bertely, 2000), el análisis realizado se hace desde un corte interpretativo (Frederick en Wittrock, 1997), todo esto se ha logrado teniendo como base la aplicación y uso de metodología propias de la investigación cualitativa orientadas y pautadas por las aportaciones de autores diversos como: Taylor (1996), Tarrés (2013). Se tuvo la postura de la observación participante desde donde se hicieron uso de instrumentos para recabar datos (diario de campo, pláticas informales), en este informe también se presentan y se comparten imágenes fotográficas ya que la mayor parte del tiempo de las clases se abocó a la elaboración de material didáctico. El análisis de los datos se presenta teniendo como respaldo teórico aportaciones de Schön (2010), Davini (2016), la intensión es reflejar escenas que evidencien estrategias que contribuyen en la formación de profesores reflexivo, pues se deben "estudiar las experiencias del aprender haciendo y el arte de una buena acción tutorial. Y debiéramos fundamentar nuestros estudios en la hipótesis de trabajo de que ambos procesos son inteligentes y -dentro de los límites aún por descubrir- comprensible se retoman aproximaciones teóricas y metodológicas de autores diversos que en su conjunto hacen aportaciones importantes para la construcción de nuevos conocimientos (Schön, 2010: 29).

La etapa de investigación de campo transcurrió a lo largo de una semana, durante la cual se sumaron dificultades y se enfrentaron (poco dominio del idioma portugués). Así como se hace camino al andar, la investigación etnográfica de aula se hace al adentrarnos al escenario, la inquietud pedagógica por aproximarme a esos otros saberes y conocimientos, en lo especifico a esas otras formas de aprender y enseñar la lectura y la escritura, desde la lengua materna a la par de estrategias didácticas diversas, pues formarse en las practicas docentes es un proceso permanente, que acompaña toda la vida profesional, pero es en la formación inicial y, sobre todo, en las prácticas de enseñanza, donde los estudiantes adquieren y desarrollan los conocimientos fundamentales de la profesión (Davini, 2016:42). 
La información y datos que se presentan en este artículo fue posible entre otras cosas, gracias a la autorización de acceso al salón de clases, donde a través de la observación participante se realizaron los registros de una semana intensiva en que se desarrolló el tema contextual ${ }^{1816}$ "Procesos de alfabetización" coordinado por la profesora Maria do Socorro Pimentel da Silva. El horario establecido es de 8:00 am a las 12:00 am y de las 14:00 horas a las 17:00 horas, tiempo en el cual se desarrollan las actividades didácticas estructuradas mediante el programa elaborado por la profesora.

El grupo es integrado por dos generaciones (turnas) de profesores y profesoras pertenecientes a diferentes grupos étnicos de los estados de Goiás, Mato Groso, Maranhão, Pará, Tocantins (ver mapa 1, para geo referenciar), como se describen a continuación.

Cuadro 1 -Alumnos de la Licenciatura en Educación Indígena (Núcleo Takinahakỹ, UFG).

\begin{tabular}{|l|l|l|l|l|}
\hline Generación & Grupos étnico & Hombres & Mujeres & Total \\
\hline $\mathbf{2 0 1 4}$ & Abtsiré & 1 & & 1 \\
\hline & Guajajara & 1 & 1 & 2 \\
\hline & T seretsu & 1 & & 1 \\
\hline & Xerente & 2 & & 2 \\
\hline & Tapirapé & 3 & & 3 \\
\hline & Tsuwaté & 1 & & 1 \\
\hline & Karaja & 2 & 3 & 5 \\
\hline & Javaé & 1 & & 1 \\
\hline & Tseredzawe & 1 & & 1 \\
\hline & Wa'omopté & 1 & & 1 \\
\hline & Dupredzamo & 1 & & 1 \\
\hline & Tsoro'rwe & 1 & & 1 \\
\hline & Tserenhowadawe & 1 & & 1 \\
\hline & Kamaiura & 1 & & 1 \\
\hline & Wadzatsé & & 1 & 1 \\
\hline & Weree & 1 & & 1 \\
\hline & & & & \\
\hline & Tsiruwe we & 1 & & 1 \\
\hline & Waura & 1 & & 1 \\
\hline & Xerente & 2 & 1 & 3 \\
\hline
\end{tabular}

18 Todo este material forma parte del archivo etnográfico, producto del trabajo de campo. 


\begin{tabular}{|l|l|c|c|c|}
\hline Generación & Grupos étnico & Hombres & Mujeres & Total \\
\hline $\mathbf{2 0 1 5}$ & Tapirapé & 1 & & 1 \\
\hline & Waura & 1 & & 1 \\
\hline Moritu & 1 & & 1 \\
\hline Karaja & & 1 & 1 \\
\hline Krahó & & 1 & 1 \\
\hline Gaviao & & 1 & 1 \\
\hline Canela & 1 & & 1 \\
\hline Krahó & & & 1 \\
\hline
\end{tabular}

Fuente: Cuadro elaborado a partir de los datos obtenidos del registro de asistencia, julio de 2017.

El grupo es integrado por profesores y profesoras de las generaciones 2014 y 2015 se aprecia también lo siguiente:

La presencia de profesoras ${ }^{1917}$ son muy pocas en relación a los varones, también lo es muy poco la representatividad de los grupos étnicos en relación a los más de 220 pueblos del Brasil, las edades son muy contrastante ya que van desde los 23 a los 55 años de edad, unos datos que no se reflejan en el cuadro (pero la información se obtuvo mediante los diálogos establecidos en el aula) es que no todos son profesores frente a grupo, algunos realizan actividades administrativas en las escuelas, existe poco dominio del segundo idioma que es el portugués, pero existe un compromiso muy fuerte con sus pueblos.

19 Uno de los datos obtenidos en función a la falta de mayor presencia de mujeres como profesoras en las aldeas de las comunidades amerindias de Brasil corresponde a supuestos como: los varones son los que tienen la responsabilidad de generar la relación con el hombre blanco para evitar posibles discriminaciones, entre los pueblos tienen la idea de que la mujer es el medio de transmisión y conservación de la lengua de la aldea, es más fácil para el hombre movilizarse para asistir a las reuniones cuando se realizan en ciudades de otros estados.... estos datos fueron proporcionados por el profesor Canela, integrante del grupo 


\section{El programa "Procesos de alfabetización ${ }^{2018 ", ~ s u ~}$ descripción}

El programa, tiene una carga de 64 horas, están dividas, 30 horas destinadas a las actividades teóricas y 24 horas a las actividades prácticas. Como base epistémica parte al establecer que: La alfabetización como inicio de un proceso más amplio de aprendizaje de la lectura y la escritura. El contexto social del niño y sus relaciones con el mundo escrito; la adquisición de lenguaje escrito a partir del mundo de los niños.

Como objetivo General establece: Posibilitar a los estudiantes un espacio de estudio y de construcción como base epistémica, a partir de un ambiente sociocultural y pedagógico que propicie la elaboración de procesos interactivos y contextuales en relación con los procesos de alfabetización de los conocimientos indígenas. Lograr lo señalado, lo prevé mediante el desarrollo de objetivos específicos como: Investigar los temas de conocimientos relevantes para la formación sociopolítica indígena, la alfabetización por medio de interacciones contextuales del mundo de los niños y desenvolver en estos procesos de aprendizaje a leer y escribir en su lengua materna, Desenvolver actividades de juegos con letras, palabras y pequeños textos, Promover actividades que retomen saberes de la oralidad.

Asimismo, el programa se sustenta al reconocer que: estudios realizados por investigadores indígenas han construido significativamente para comprender como es la educación de los niños en las sociedades indígenas. Esa educación se basa a partir de formas como cada comunidad piensa y organiza la vida colectiva, así como la formación de la ciudadanía, que normalmente está orientada con ejemplos...etc. con base a esas referencias se integran los temas a considerar que son: Ciclo de formación de personas, Pedagogía indígena, Palabras contextualizadas, Aprender a leer conociendo el territorio, juegos y actividades, Jugando con letras y formando palabras del medio ambiente.

La Metodología a desarrollar se sustenta en contexto de los alumnos, partiendo de la realidad, de que, la alfabetización de los conocimientos indígenas reivindica la adopción de metodologías contextuales, las cuales permiten trabajar los conocimientos por

20 Los datos que se presentan son extraídos del programa diseñado por la profesora. 
medio de temas que forman parte de lo cotidiano de los niños indígenas, aprovechando todas las sabidurías que se hacen en varios espacios comunitarios en los que ellos conviven y aprenden. A partir de esas referencias, serán promovidas actividades de lectura y escritura por medio de juegos, música, es importante percibir la escuela como un espacio para que los niños vivan como un medio para desenvolver los aprendizajes de alfabetización. Otro de los elementos en que integran el programa es la evaluación, para ello considera la participación en clases, la producción individual y en grupo, el desarrollo de actividades propuestas, creatividad pedagógica relacionada con la práctica docente y el conocimiento de estudio.

Por último, cerramos este apartado señalando que la enseñanza es una actividad intelectual que involucra complejas situaciones y decisiones, en donde además se tienen que echar a mano y poner en prácticas diversas estrategias didácticas que propicien el logro de los aprendizajes, y a su vez, la reflexión crítica en los escolares en vía de la formación docente.

\section{Desarrollo de una práctica docente}

La clase inicio con el pase de lista a cargo de la asistente de la profesora (actividad que se realizó todos los días de la semana que abarco el curso y en ambas jornadas, mañana y tarde), seguidamente la profesora después de dar la bienvenida al curso y de mi integración al grupo, mostro el juego de mesa "la lotería mexicana"2119, luego

21 El juego de la lotería, ampliamente difundido en México, tiene su origen en las Ferias mexicanas durante las décadas de los 50 del siglo XIX, es un juego de azar que consta de 54 cartas y un número indefinido de tarjetas llamadas "tablas" con 16 imágenes de las cartas escogidas aleatoriamente. Cada vez que se extraiga una carta del grupo, ésta se anuncia, suele usarse un verso (hay muerte, que vienes a ser, si se muere mi mujer me caso con mi cuñada, "LA MUERTE"), los participantes deben marcar esa carta en sus tablas si la tienen. El ganador será quien primero forme en su tabla la alineación que se haya especificado al inicio del juego con las cartas marcadas y grite "lotería", aunque la lotería se acostumbra a jugar en lugares públicos (ferias, kermeses, festivales escolares, etc.), también es un popular pasatiempo casero, por lo que resulta muy sencillo encontrar a la venta este juego en mercados públicos, papelerías o tiendas de autoservicio. Incluso existen variantes en que las imágenes son adaptadas en referencia a alguna ocasión especial... 
de ello, empezó a explicar algunas normas del juego (de manera general) georeferenciando la procedencia, la profesora solicito que participara para compartir algunas otras características del juego y las reglas, mientras tanto, ella y su asistente distribuía los tableros y pidiendo al grupo que observaran bien el contenido, agregó, "así como ese tipo de juego de mesa, existen otros juegos con temáticas específicas: animales domésticos, números, frutas, flores, animales acuáticos, etc... que pueden generar interés a los niños, porque la manera en que se juegan resultan ser divertidas".

Continuó diciendo, que estaría bien organizar y hacer algo parecido como un recurso más de los materiales didácticos para ofertar a los escolares en la etapa del aprendizaje de la lectura y escritura de sus comunidades, pues en el proceso de la alfabetización es necesario, echar mano de muchas estrategias, para ello solicito que se integraran en equipos de acuerdo al idioma, para hacer más cómodo el trabajo.

La actividad docente realizada, permite argumentar lo que indica Davini $(2016,47)$, "Es evidente que quienes enseñan necesitan saber o tener un relativo dominio sobre el conocimiento, la habilidad o experiencia que desean trasmitir. Pero conocer o poseer este saber no es suficiente: se necesita también pensar para qué enseñarlo, cómo enseñarlo y tomar decisiones..." entre otras cosas. La profesora sabe hacia dónde dirige su enseñanza, basada de los objetivos de su programa, encauzo al grupo a construir juntos estrategias didácticas, desde los contextos sociales y culturales de cada profesor, con ello, abordar el tema de la alfabetización y aprender como se generan los procesos del aprendizaje.

Los estudiantes se organizaron, la profesora a través de la asistente les proporcionó material didáctico (cartulinas, reglas, tijeras, marcadores, crayolas, colores, entre otros), los equipos de trabajo tomaron el material pertinente, dialogaron para acordar sobre que temática realizarían el material didáctico, las normas del juego y la finalidad, las actividades permitieron escuchar que los maestros externaron las diferentes fuentes de conocimiento cotidiano relacionado con las prácticas culturales de sus pueblos, y que seguramente también y como lo aborda Mercado "provenientes de diversos contextos experimentales que ellos articulaban en torno a sus problemas de enseñanza" $(2012,37)$. Al mismo tiempo la profesora pasaba a cada equipo para saber sobre las decisiones y acuerdos tomados por cada los grupos. (ver imagen 1). 
El texto planteado, da elementos y permite argumentar con solidez, para anunciar que en algunas situaciones "Los estudiantes aprenden mediante la práctica de hacer o ejecutar aquello en lo que buscan convertirse en expertos, y se les ayuda a hacerlo así por medio de otros prácticos más veteranos... les inician en las prácticas en las tradiciones de las prácticas" (Schön, 2010: 29).

La profesora reconoce que cada profesor porta un estilo pedagógico propio, que lo hace único en los procesos de la enseñanza y del aprendizaje de la alfabetización (ver imagen 1), no descarta la idea que en la formación y desarrollo de esta habilidad y conocimiento es determinada en parte por las situaciones diversas por la que atraviesa la escuela, a través de los factores externos (la precarias situaciones económicas, los avances y usos de la tecnología en contraposición de la riqueza cultura las comunidades, considerando la relación hombre - naturaleza). En este sentido, es importante tener presente que "No se puede enseñar al estudiante lo que necesita saber, pero puede guiársele" (Schön, 2010: 29), a tal grado que cada estudiante vaya descubriendo a leer la direccionalidad que señala la brújula de acuerdo a lo que precisa lograr.

Imagen 1 - Integración de equipos de trabajo de acuerdo al idioma de los integrantes del aula.

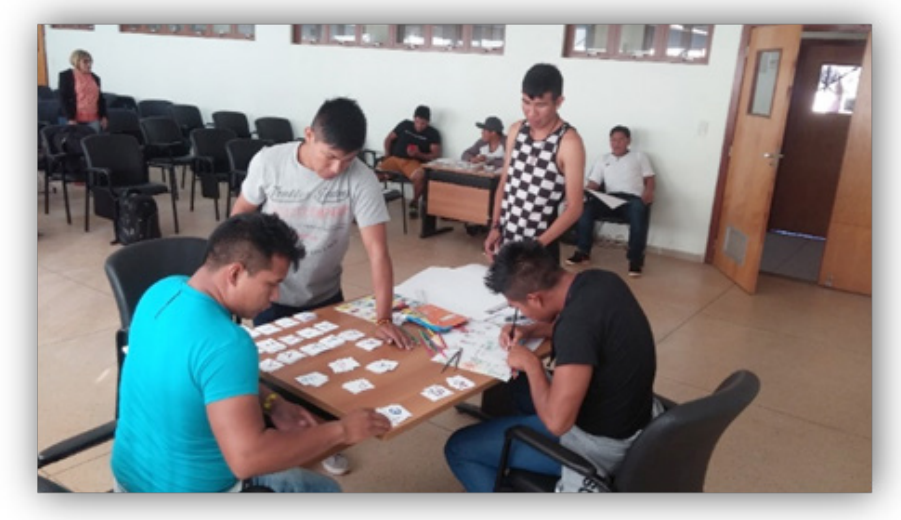

Fuente: Propia, profesores elaborando sus cartillas como material didáctico para la enseñanza de la lectura y escritura en sus escuelas. 17 de julio de 2017, UFG, Goiânia, Brasil.

Los profesores organizados en grupos poco a poco fueron desarrollando la actividad acordada, aunque partieron del ejemplo de 
la "lotería mexicana" cada equipo tenía un diseño propio. "el alumno tiene que ver por sí mismo y a su propia manera las relaciones entre los medios y los métodos empleados y los resultados conseguidos. Nadie más puede ver por él, y no puede verlo simplemente porque alguien se lo diga, aunque la forma correcta de decirlo pueda orientar su percepción para verlo y así ayudarle a ver lo que necesita ver" (Schön, 2010: 151).

Una de las características del desarrollo de la práctica docente fue, que la profesora no estableció, ni señalo tiempo para la conclusión de la actividad, cada grupo trabajó a su ritmo, teniendo en cuenta que debían concluir en un tiempo considerado ${ }^{2220}$, por lo que tener prejucio con el tiempo, terminaron sus materiales didácticos, fueron dos las sesiones que ocuparon en sus dos turno, mañana y tarde, la profesora estuvo siempre dando acompañamiento y preguntando sobre los las estrategias a realizar con los escolares para la socialización del material y el acercamiento de los escolares al letramento (aprendizaje de la escritura y la lectura).

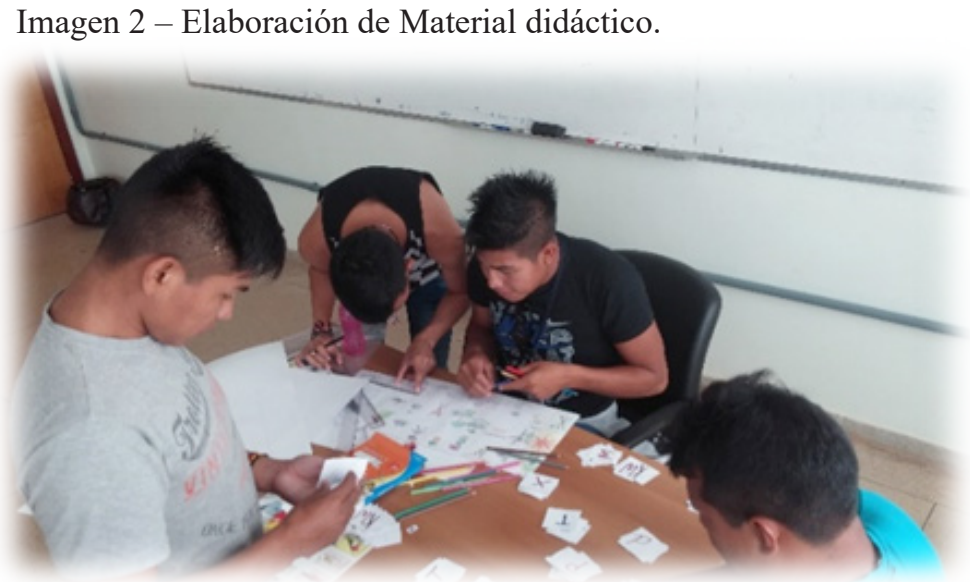

Fuente: Propia. 18, Julio de 2017, UFG, Goiânia, Brasil.

22 Esta acción permite pensar que puede ser un reflejo de la actitud que promueven los profesores en sus centros escolares hacia y con sus alumnos, además una de las características de los profesores originarios de Brasil, en ciertos aprendizajes, el tiempo no se mide, factor que en las escuelas de educación básica generales se aplica como disciplina, a los escolares se les presiona en la elaboración de sus actividades. 
En la tercera sesión, la profesora abordo el programa del tema, haciendo énfasis en algunos puntos sobre la alfabetización entre los que destacó:

En los procesos de enseñanza y del aprendizaje de la lectura y de la escritura es necesario tener en cuenta factores que intervienen favoreciendo en el escolar el aprendizaje, por lo cual es necesario que se consideren prioritarios, estos son las actividades cotidianas de cada pueblo, por lo que se deben partir de esas experiencias vividas y de interés del escolar.

En las diversas comunidades, aunque existen estratificaciones de edades, esto no es un factor que impida a los niños involucrarse en las diversas actividades comunitarias (Maybury-Lewis, 1974) por lo que en los procesos del aprendizaje de la lectura y la escritura (letramento) se deben considerar las acciones (ceremonias, rituales, etc.) más familiares como temáticas para el acercamiento a los procesos de la alfabetización. Cuando los temas son de pertinencia cultural, promueven diálogos entre las voces presentes, que rescatan amigablemente los ausentes, vitalizando, asimismo, los saberes milenarios" (Pimentel da Silva, 2017: 208).

En la sesión de la tarde, profesora propicio la participación de los alumnos a socializar algunas de las practicas cotidianas de sus comunidades, al concluir esta fase, los profesores continuaron con sus actividades didácticas, "las cartillas".

Siguiendo con la temática, el cuarto día de sesión, la profesora dio las instrucciones para que cada grupo pasara a socializar sus actividades realizadas:

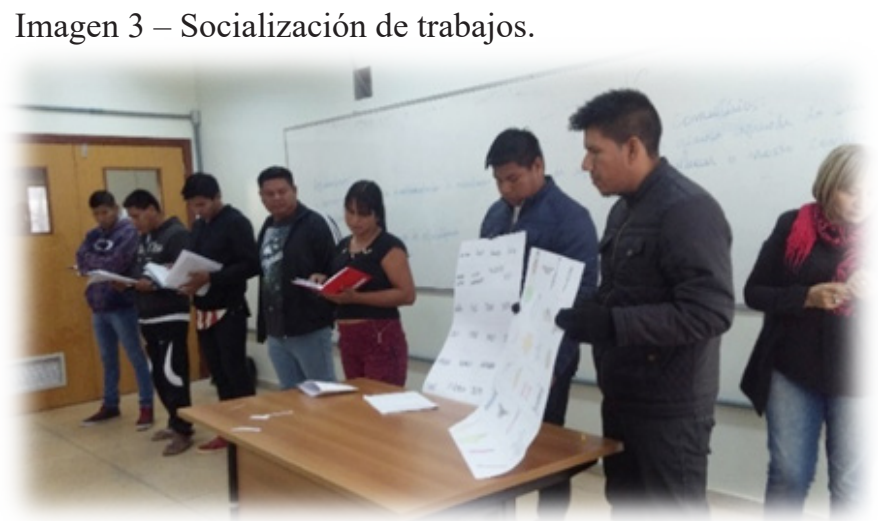

Fuente: Propia. 19, Julio de 2017, UFG, Goiânia, Brasil. 
Al siguiente día, al concluir las presentaciones de los trabajos de las cartillas didácticas, la profesora propuso realizar "una sopa de letras", para ello pidió que todos los que habían realizado tarjetas con letras, las depositaran en un solo lugar, para hacer un banco de letras, posteriormente la profesora externó que ella diría las palabras y los integrantes de grupos las formarían con las tarjetas; las palabras fueron las siguientes: sol, luna, nube, agua, casa, árbol, paloma.

Los integrantes de cada grupo se coordinaban para formar las palabras, ya que se tenía que seleccionar las tarjetas. La profesora externó que, así como cada uno de los integrantes del grupo se involucraba a la actividad didáctica, a los escolares también se les hace interesante estos tipos de actividades, ya que se parten de los saberes y del contexto de la niña y del niño. (Ver imagen 5). La situación didáctica también permite argumentar que la concepción del objeto de enseñanza en función de los aportes lingüísticos y la concepción del sujeto que aprende a leer y escribir...es una condición importante para contribuir, al cambio de la propuesta didáctica en se vive en muchas de las escuelas (Lerner, 2004:64).

Imagen 5 - Formando palabras, "material didáctico".

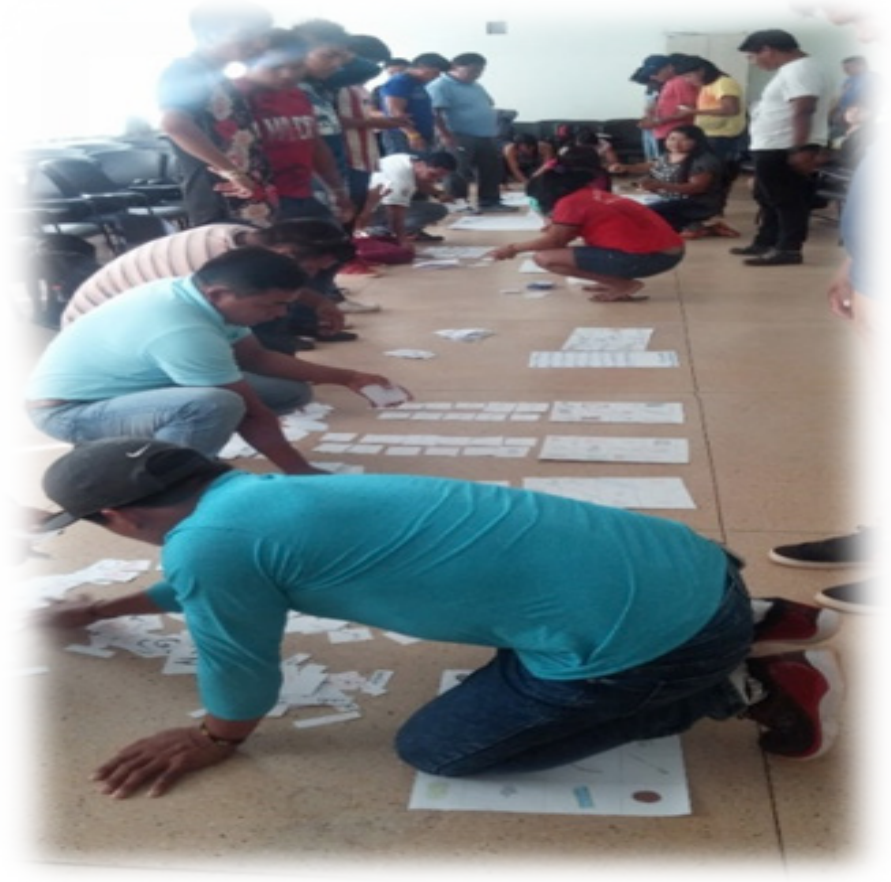

Fuente: Propia. Julio de 2017, UFG, Goiânia, Brasil. 


\section{Una forma propia del proceso de alfabetización: reflexiones finales}

Las experiencias compartidas por los profesores que asistieron al tema contextual "Procesos de alfabetización", son testimonios que permiten plantear que los docentes se convierten en sujetos únicos y verdaderos al conducir el rumbo y sentido pedagógico de la educación en sus comunidades, en ellos recae una gran responsabilidad, como lo es la alfabetización (la enseñanza de la lectura y escritura), que se aborda mediante, las acciones pedagógicas que emprenden, mismas que determinan la seguridad y orientación de sus pueblos, en tanto sus acciones, en teoría y práctica son coherentes con la realidad epistemológica en la que interactúa $\mathrm{n}$, sus aportaciones son lecciones que se deben tomar como ejemplo para ser socializadas, pues, "Se puede aprender mucho de quienes son capaces de hacer cosas en los contextos reales de la práctica" (Davini, 2016: 20).

La convivencia pedagógica $\mathrm{y}$ actividades didácticas derivadas de los encuentros, cuando se tiene un fin común, permiten evidenciar que los procesos para el aprendizaje y la enseñanza de la alfabetización se enrique aún más cuando se construyen juntos, pues las participaciones en donde permitieron conocer las experiencias de cada uno de los integrantes desde sus contextos contribuyo a la construcción de nuevas actividades didácticas, esas prácticas docentes son un derrotero que permite ampliar el abanico de estrategias didácticas para favorecer el aprendizaje y la enseñanza de la lectura y de la escritura, (la alfabetización).

A través de la convivencia y el desarrollo del tema contextual "Procesos de alfabetización" se puede predecir que los procesos de alfabetización en las niñas y niños de los diferentes grupos étnicos brasileiros se inician y son pautadas teniendo en cuenta las prácticas culturales de cada pueblo, la relación con la naturaleza, la cosmovisión, en los saberes de lo cotidiano, por ejemplo: el canto, los juegos infantiles, las narraciones (cuentos, leyendas e historias de vida), pero además, las formas de abordarlas se convierten en didácticas pedagógicas propias, los profesores parten de ellas con la finalidad de perpetuar y trasmitir a las nuevas generaciones la riqueza de los saberes y de la cultura de cada pueblo para pasar de ello al aprendizaje de la lectura y la escritura, la alfabetización. 
Los procesos del aprendizaje de la lectura y de la escritura no parte del supuesto teórico derivado de la psicogenésis de la escritura, planteado por investigadoras citadas, en estos contextos no se da mayor importancia al desarrollado logrado del infante respecto a los niveles conceptuales de la escritura, lo que se le apuesta con mayor énfasis, es al interés que van desarrollando los escolares respecto a los temas abordados que les permitirá alfabetizarse sobre la temática, por consiguiente, comprender la relación y necesidad de hacer uso de la lengua escrita y de la lectura.

Las construcciones sociales colectivas de los profesores son desarrolladas en la dimensión de la vida cotidiana ya evidencia líneas de pensamientos proveniente de distintos ámbitos culturales de las comunidades, lo expresado por los profesores es producto de su pensamiento construido a partir de su cosmovisión, la oportunidad como observador participante permite externar que la trayectoria de formación docente también se construyen teniendo como base el saber cotidiano sobre la enseñanza en determinados contextos definidos como situaciones particulares, lo que da pauta a considerar que es necesario

continuar produciendo conocimientos que permitan resolver los múltiples problemas que la enseñanza de la lengua escrita plantea, y de hacerlo a través de estudios cada vez más rigurosos, de tal modo que la didáctica de la lectura y la escritura deje de ser materia "opinable" para constituirse en un cuerpo conocimiento de reconocida validez (Lerner, 2004, p. 67).

Los principios didácticos, fueron evidentes mediante las actividades didácticas realizadas por la profesora, los cuales encauzaron los intereses de los estudiantes, los que a partir de sus experiencias en función de sus contextos socioculturales se involucraron para construir juntos otras estrategias didácticas que los encamino a la comprensión del proceso de la alfabetización, en este tenor fue evidente el logro de los objetivos establecidos en el programa del tema, como bien lo señala Davini (2016, p. 48), la necesidad de formar docentes capaces de reflexionar y de elaborar alternativas, fortaleciendo su juicio y su papel de profesional. 


\section{Fuentes de consultas}

BARBOSA, H. (1968). Cómo han aprendido a leer y escribir los mexicanos. México: Galve.

BERTELY, M. (2000). Conociendo nuestras escuelas, un acercamiento etnográfico a la cultura escolar. México: Paidós.

DAVINI, M. (2016). La formación docente en la práctica docente. Argentina: Paidós.

FERREIRO, E.; TEBEROSKY, A. (1979). Los sistemas de escritura en el desarrollo del niño. México: S. XXI.

FERREIRO, E.; RODRÍGUEZ, B. (1994). Las condiciones de alfabetización en el medio rural. México: CINVESTAV.

FERREIRO, E. (2007). Alfabetización de niños y adultos: textos escogidos. México: CREFAL.

. (1997). Alfabetización teoría y práctica. México: S.XXI.

FERREIRO, E.; GÓMEZ, M. (1982). Análisis de las perturbaciones en el proceso de aprendizaje de la lecto-escritura. México: Dirección General de Educación Especial, SEP - OEA.

FREIRE, P. (2016). El maestro sin receta. México: S. XXI.

. (2013). Por una pedagogía de la pregunta. México: S. XXI.

. (2006). La importancia del acto de leer. Venezuela: Laboratorio educativo.

. (2003). Por una pedagogía de la autonomía. México: S. XXI.

. (1994), Cartas a quien pretende enseñar. México: S. XXI.

LERNER, D. (2004). Leer y escribir en la escuela. México: SEP.

MAYBURY, D. (1974). Akwé Shavante Society. Brasil: Libraria Francisco Alves

MERCADO, R. (2002). Los saberes docentes como construcción social. La enseñanza centrada en los niños. México: FCE.

MORÍN, E. (2008). La cabeza bien puesta. Argentina: Nueva Visión. . (2002). Educar en la era planetaria. España: Gedisa. 
OLMOS, A.; CARRILLO, A. (2009), Juegos y alfabetización. México: UNAM.

PIMENTEL DA SILVA, M. S. (2017). A pedagogia da retomada: decolonização de saberes. En: Articulando e Construindo Saberes. Saberes indígenas na escola. Vol. 2, N. 1. Brasil: UFG. pp. 203 -215.

. (2016). Alfabetização pelos conhecimentos indígenas: reflexões sobre suas possibilidades e limitações. En: Saberes indígenas na escola. Vol. 1, N. 1. Brasil: UFG. pp. 15 - 20.

. (2016). Diversidad cultural indigena brasileira e reflexões no contexto de educação básica. Brasil: Espaço Acadêmico.

RINCÓN, C. (2003). La enseñanza de la lectura y de la escritura en Colombia. Colombia: Cooperativa editorial magisterio.

RODRÍGUEZ, I. (2017). Enseñanza inicial de la lengua escrita: cuándo iniciar la enseñanza y cómo hacerlo. Una reflexión desde las creencias de los docentes en: Perfiles educativos tercera época Volumen XXXIX número 156.

SANTOS, L. (2006). O Índio Brasileiro o que vocé precisa saber sobre os povos indigenas no Brasil de hoje. Brasil: MEC/UNESCO.

SCHÖN, D. (2010). La formación de profesionales reflexivos, hacia un nuevo diseño de la enseñanza y el aprendizaje en las profesiones. España: Paidós.

SOARES, M. (1989). Alfabetização no Brasil: O Estado do Conhecimento. Brasil: Inep/Reduc.

. (1986). Linguagem e Escola: Uma Perspectiva Social. Brasil: Ática.

TARRÉS, M. (2013). Observar, escuchar y comprender sobre la tradición cualitativa en la investigación social. México: El Colegio de México.

TAYLOR, S. J. Y R. B. (1990). Introducción a los métodos cualitativos de investigación. Argentina: Paidós.

TOABADA, A. (1997). Colonialismo en la educación: ¿hasta dónde pueden llegar sus consecuencias? En: Propuesta educativa. Año 8 No. 17, Argentina. pp. $56-58$.

STRAUSS, A.; CORBIN, J. (2002). Bases de la investigación. Técnicas y procedimientos para desarrollar la teoría fundamentada. Colombia: Universidad de Antoquia.

VIGOTSKY, L. (1978). El desarrollo de los procesos psicológicos superiores. España: Crítica y Aprendizaje. 
. (1988). Pensamiento y lenguaje. México: Quinto Sol.

. (1978). Psicología y pedagogía. Argentina: Aíque.

WITTROCK, M. (1997). La investigación de la enseñanza II. España: Paidós.

\title{
Anexo Galería de actividades académicas
}

\begin{abstract}
Material didático
\end{abstract}

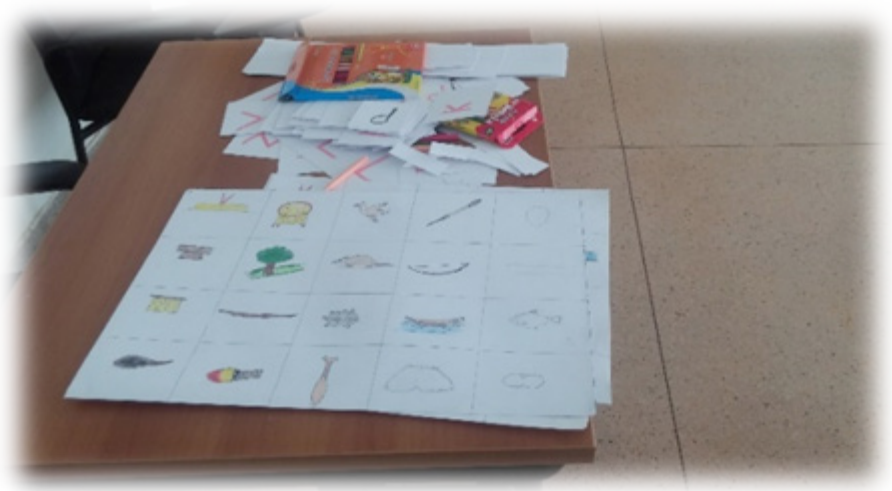

Fuente: Propia. Julio de 2017, UFG, Núcleo Goiânia, Brasil.

Socialización de trabajos

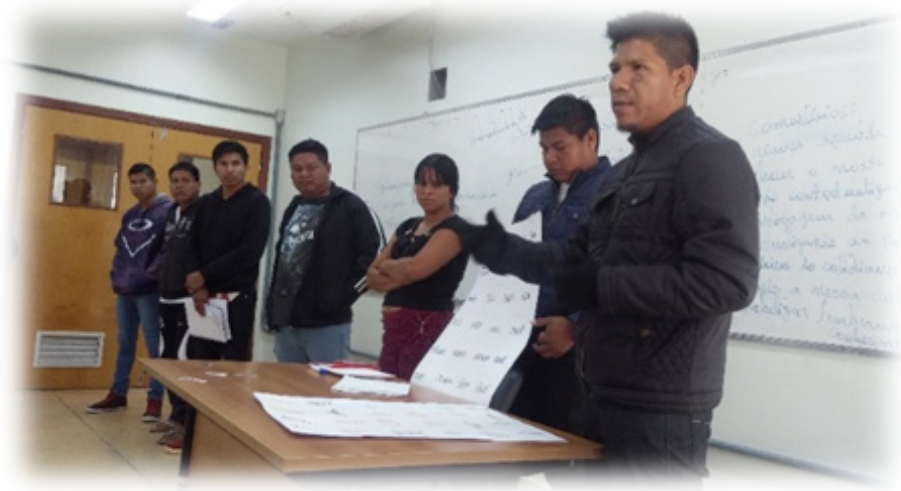

Fuente: Propia. Julio de 2017, Goiânia, Brasil 
Socialización de trabajos

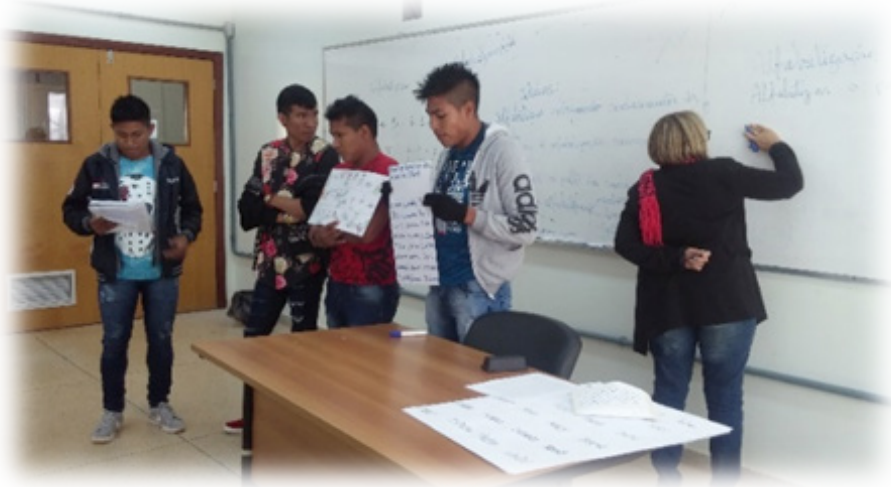

Fuente: Propia. Julio de 2017, Goiânia, Brasil

Socialización de trabajos

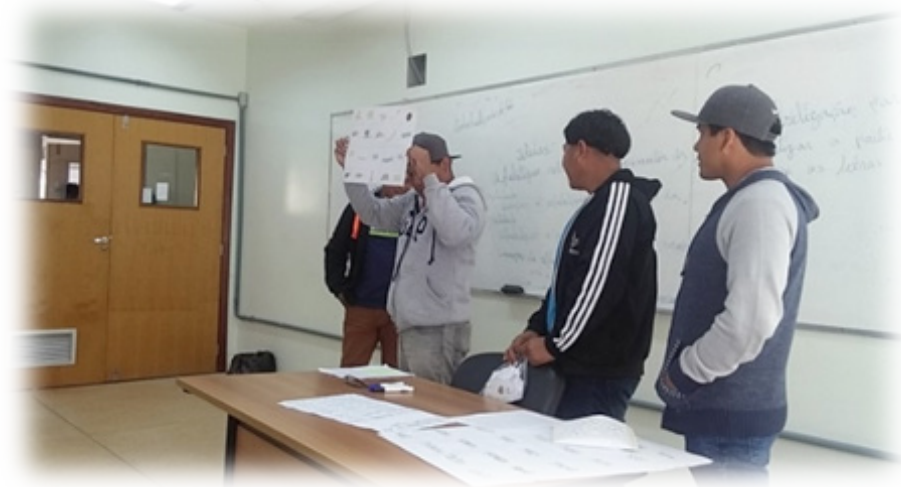

Fuente: Propia. Julio de 2017, Goiânia, Brasil.

Socialización de trabajos

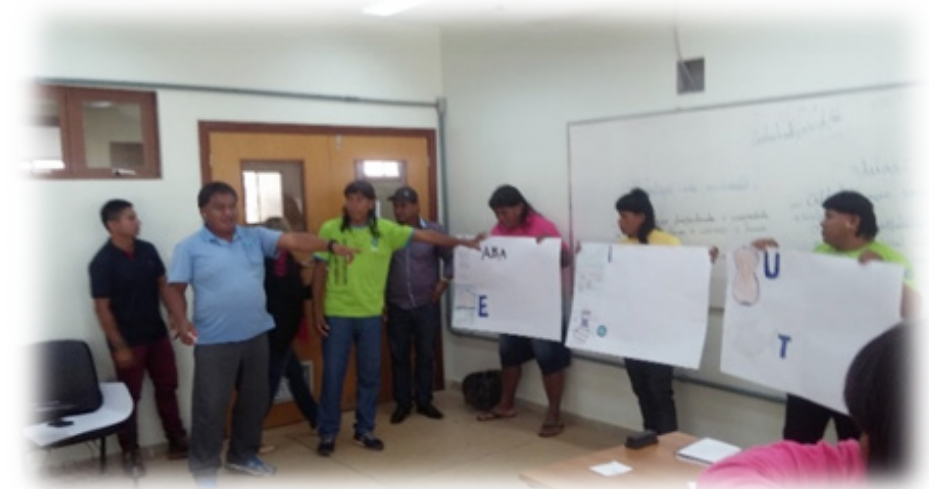

Fuente: Propia. Julio de 2017, Goiânia, Brasil. 
Socialización de trabajos

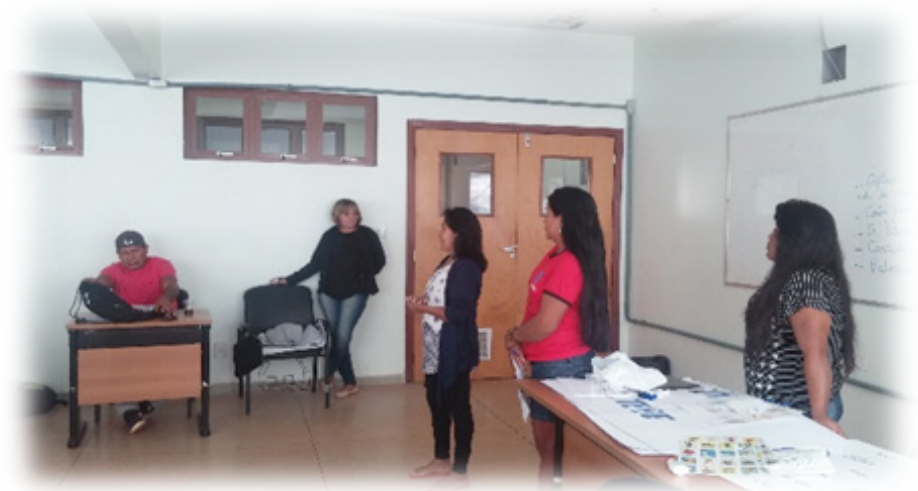

Fuente: Propia. Julio de 2017, Goiânia, Brasil.

Recebido para publicação em maio de 2018. Aceito para publicação em junho de 2018. 